\title{
Experimental Demonstration of an All-Optical 2R Regenerator with Adjustable Decision Threshold and "True" Regeneration Characteristics
}

\author{
Geert Morthier, Mingshan Zhao, Bart Vanderhaegen, and Roel Baets
}

\begin{abstract}
Experimental $2 \mathrm{R}$ regeneration characteristics of an Optical Decision Circuit based on a Mach-Zehnder Interferometer with Gain Clamped Semiconductor Optical Amplifiers in both arms are reported for the first time. The decision circuit gives a true regeneration, i.e., a digital characteristic on a linear instead of a logarithmic (or $\mathrm{dB}$ ) scale for the input and/or output power.
\end{abstract}

Index Terms-Optical communication, optical pulse shaping, semiconductor optical amplifiers.

\section{INTRODUCTION}

I T IS now generally accepted that regeneration, although not necessarily all-optical, will be indispensable in long fiber links in order to remove the noise, crosstalk, and signal distortion that have accumulated during signal propagation. Such regeneration can consist of simply amplifying the signals, but will more likely also include signal reshaping ( $2 \mathrm{R}$ regeneration) and retiming ( $3 R$ regeneration) for very high bitrate signals. At high bitrates, an all-optical regeneration ([1]-[4]) may be preferred over an opto-electronic one [5] because of cost and complexity advantages. Since $3 R$ regenerators consist essentially of a $2 R$ regenerator and a clock extraction system, $2 \mathrm{R}$ regenerators, also called optical decision circuits, can be expected to be of great importance in the future.

New components or subsystems for optical regeneration (both $2 \mathrm{R}$ and $3 \mathrm{R}$ ) have been proposed several years ago. The semiconductor components have some advantages over the fiber-based regenerators because of their compactness and their potential for low-cost production. More recently, several new semiconductor-based $2 \mathrm{R}$ regenerators have been proposed. Thereby, attention has focused on the regenerative capabilities of all-optical interferometric wavelength converters (e.g., [1], [2]), on $Q$-switched DFB lasers [3], and on an SOA-based interferometric Michelson structure [4]. However, regeneration based on wavelength converters has the disadvantage of requiring a pump laser. In some cases (although not always), the fact that a new signal is generated (of which some properties may be different) may also be undesirable. In addition, the decision threshold is fixed and determined by the structure and, therefore, can not be modified to take into account the

\footnotetext{
Manuscript received February 1, 2000; revised May 23, 2000. This work was supported by the ACTS project REPEAT (AC-305).

The authors are with the Department of Information Technology, University of Gent - IMEC Sint-Pietersnieuwstraat 41, B-9000 Gent, Belgium (e-mail: morthier@intec.rug.ac.be; mszhao@intec.rug.ac.be; baets@intec.rug.ac.be; bvdhaeg@rug.ac.be).

Publisher Item Identifier S 1041-1135(00)09567-7.
}

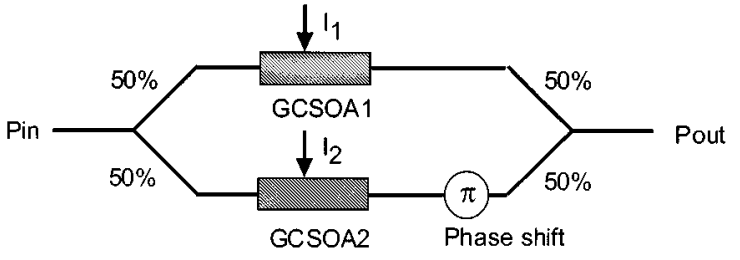

Fig. 1. Symmetrical MZI-based implementation of an optical decision circuit.

properties of the incoming signal. The regenerators based on $Q$-switched lasers also are based on the generation of a new signal. Moreover, their decision characteristics exhibit a hysteresis and the decision threshold can not be modified in a straightforward manner. The SOA-based interferometric structure presented in [4] does not possess any of the aforementioned disadvantages, but it also does not give a true digital characteristic. This decision characteristic has so far only been presented on a logarithmic scale, and careful inspection reveals that it gives some regeneration at high power levels (i.e., for the ones) but little or no regeneration at low power levels (for the zeros).

A $2 \mathrm{R}$ regenerator which gives a true decision characteristic (i.e., when linear instead of $\mathrm{dB}$ scales are used for both input and output power), of which the decision threshold and the decision slope can be easily (electronically) adjusted, and which does not require a pump laser has been proposed earlier by the authors [6]. However, so far only simulation results for this new regenerator device have been presented. Here we present the first experimental results, which clearly demonstrate the above mentioned properties. The structure of the component and its operation principle are briefly explained in Section II. Section III then discusses the measured regeneration characteristics and proposes some further improvements, including some tricks that may make this component suitable for bitrates of $10 \mathrm{Gbit} / \mathrm{s}$ or even multiples of that bitrate. It is emphasized that the component can, in principle, be fabricated as a photonic integrated circuit, although here we report results on a hybrid fiber-based device.

\section{BASic StRUcture AND Operation PRINCIPLE}

The Mach-Zehnder interferometer (MZI)-based circuit that is used as a $2 \mathrm{R}$ regenerator, and which was proposed in [6] with symmetric splitters and identical gain clamped semiconductor optical Amplifiers (GCSOAs) in both arms, is shown in Fig. 1. The GCSOAs are travelling wave amplifiers implemented as DBR lasers with Bragg sections on either side of the 


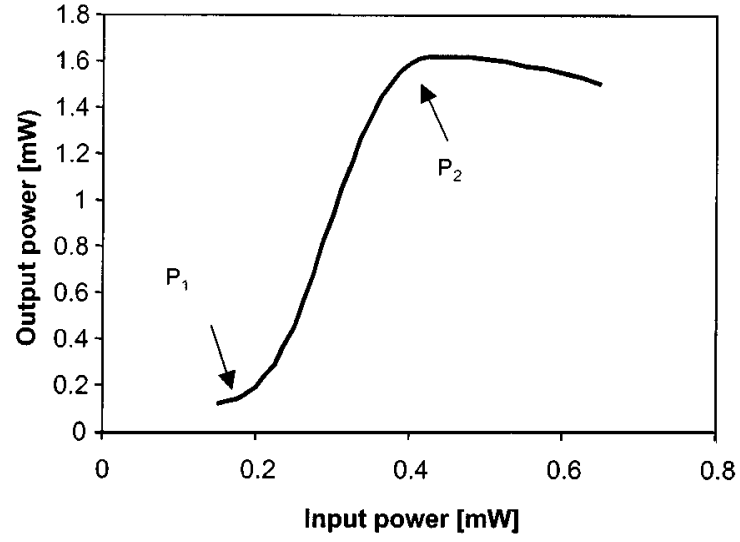

Fig. 2. Experimental regeneration characteristic of a fiber- optic implementation of the regenerator in Fig. 1.

active section and with AR-coating on both facets. Both amplifiers in Fig. 1 are assumed to have a different bias current such that they exhibit equal amplification and phase shift below the saturation power and a different saturation power. It is emphasized once more that an identical amplification for different saturation powers can only be obtained with GCSOAs and not with ordinary travelling wave amplifiers.

The phase difference of $\pi$ between both interferometer arms implies that a perfect destructive interference (hence, zero output power) is obtained, as long as both amplifiers have equal gain (i.e., as long as none of them is saturated). On the other hand, if both amplifiers are saturated, they both give a different, yet input power independent, output power and the interference results in a constant, nonzero output power at the output of the MZI.

For our experiments, we built a fiber-optic MZI with two considerably different GCSOAs. One of the GCSOAs is a packaged GCSOA with built-in temperature control, while the other GCSOA is a bare chip. The temperature control in the packaged GCSOA was used to obtain the desired phase relationship between both interferometer arms, while the in and out coupling of the bare GCSOA was used to obtain equal amplification in both arms. The complete set- up is covered by a plastic box to avoid output power instabilities due to air flow. The lasing wavelength of the GCSOAs is around $1510 \mathrm{~nm}$, while the signal wavelength is around $1550 \mathrm{~nm}$.

\section{EXPERIMENTAL CHARACTERISTICS OF THE REGENERATOR AND DISCUSSION}

Fig. 2 shows the static regeneration characteristic that was found experimentally for a current of $I_{1}=72 \mathrm{~mA}$ through the GCSOA chip and of $I_{2}=54 \mathrm{~mA}$ through the packaged GCSOA. Notice that in our set-up, the packaged GCSOA has the highest saturation output power of the two GCSOAs although it has the smallest current. An extinction ratio of $12 \mathrm{~dB}$ is obtained for the regeneration characteristic. This rather moderate extinction ratio is due to the difference of about $2 \mathrm{~dB}$ in the fiber-to-fiber gain of both GCSOAs in the set-up. This results in an imperfect destructive interference and hence a nonnegligible output power level for small input powers. Also, the state of polarization can differ slightly between the two interfering

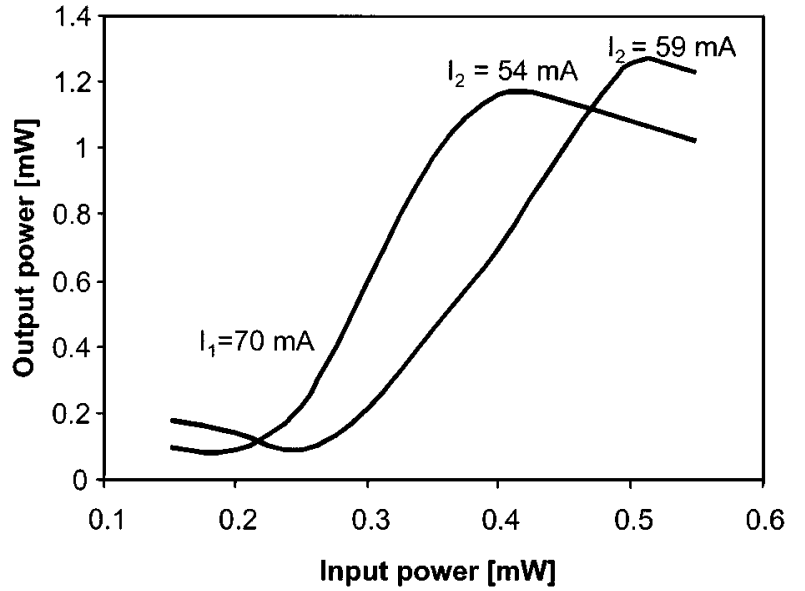

Fig. 3. Change of the regeneration characteristic with changing bias current.

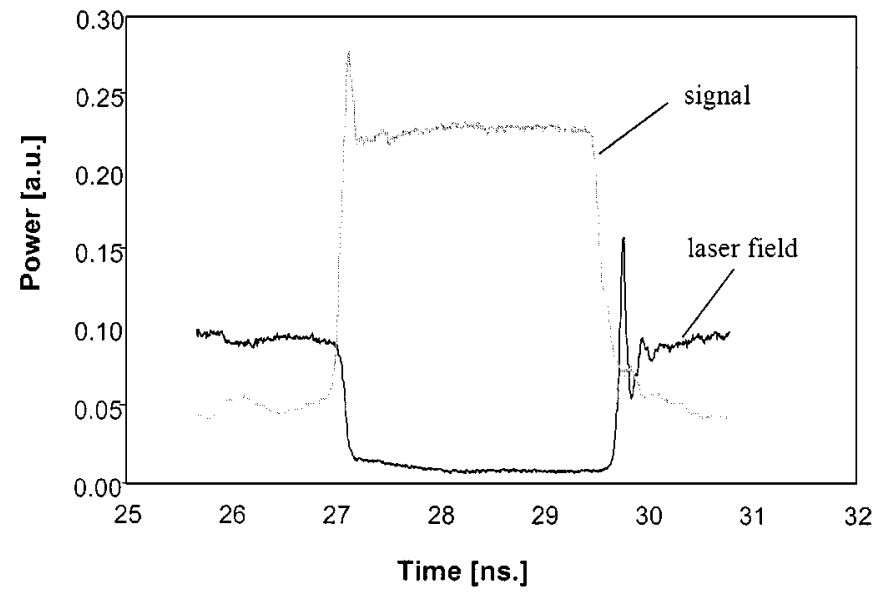

Fig. 4. Transient response of laser field and signal output power for a GCSOA (driven at $100 \mathrm{~mA}$ ) and for rectangular input pulses with 200-MHz repetition rate and an extinction ratio of $10 \mathrm{~dB}$

fields after propagating through the spacious device. For these reasons, we believe that an integrated version of the regenerator would show improved extinction ratio.

The points $P_{1}$ and $P_{2}$ in Fig. 2 depend on the bias currents $I_{1}$ and $I_{2}$ of the two GCSOAs respectively. Changing one or both of the bias currents would allow the adjustment of the decision threshold and/or the slope of the decision characteristic. This is illustrated in Fig. 3 which shows that the decision characteristics for two different values of the bias current $I_{2}$ clearly exhibit a different slope.

The regeneration characteristics have not been measured under dynamic conditions and, therefore, it is only possible to give some considerations about the maximum bitrate. In [6], it was found that the relaxation oscillations occurring during the on- and off-switching of the laser mode in the GCSOAs seriously affect the transient behavior of the regenerator. This on- and off-switching corresponds with the amplifier going out and into saturation. Time domain simulations, shown in [6], indicated that the maximum bitrate was limited to about 3 Gbit/s. Preliminary measurements of the dynamic behavior of a single GCSOA, with an input consisting of periodic pulses driving the amplifier into saturation, confirm this prediction. The measurement results, shown in Fig. 4 for a GCSOA chip 
biased at $100 \mathrm{~mA}$, clearly show relaxation oscillations with a resonance frequency of around $5 \mathrm{GHz}$ and a total duration of about 0.4 ns. However, these measurement results have to be contrasted with the recent discovery that DBR lasers can give very high bandwidths (e.g., $30 \mathrm{GHz}$ ) and very high resonance frequencies [7]. If appropriately designed, even higher resonance frequencies should be possible in DBR-based GCSOAs since they lase at wavelengths with large detuning from the gain peak and very high differential gain. With such amplifiers, the regenerator proposed here should easily be capable of operation at $10 \mathrm{Gbit} / \mathrm{s}$ or at multiples of that bitrate (probably even up to $40 \mathrm{Gbit} / \mathrm{s}$ ).

\section{CONCLUSION}

We have presented experimental characteristics of an earlier proposed $2 \mathrm{R}$ regenerator. The characteristics confirm the earlier theoretical prediction, i.e., excellent regeneration is obtained on a linear scale and the decision threshold and slope are easily and electronically adjustable. Also, possible improvements and an assessment of the maximum usable bitrate have been given.

\section{REFERENCES}

[1] B. Mikkelsen, S. L. Danielsen, C. Joergensen, R. J. S. Pedersen, H. N. Poulsen, and K. E. Stubkjaer, "All-optical noise reduction capability of interferometric wavelength converters," Electron. Lett., vol. 32, pp. 566-567, 1996.

[2] B. Lavigne, D. Chiaroni, L. Hamon, C. Janz, and A. Jourdan, "Experimental analysis of SOA based $2 \mathrm{R}$ and $3 \mathrm{R}$ optical regeneration for future WDM networks," in Proc. Opt. Fiber Comm. Conf. 98, San Jose, USA, paper Th07.

[3] B. Sartorius and M. Mohrle, "Mirror modulated lasers: A concept for high speed transmitters," Electron. Lett., vol. 32, pp. 1781-1782, 1996.

[4] D. Wolfson, P. B. Hansen, A. Kloch, and K. E. Stubkjaer, "All-optical $2 \mathrm{R}$ regeneration based on interferometric structure incorporating semiconductor optical amplifiers," Electron. Lett., vol. 35, pp. 59-60, 1999.

[5] P. Ohlen, L. Thylen, and E. Berglind, "Dispersion limits in $10 \mathrm{~Gb} / \mathrm{s}$ standard fiber systems using nonlinear optoelectronic repeaters," IEEE Photon. Technol. Lett., vol. 9, pp. 1155-1157, Aug. 1997.

[6] G. Morthier, J. Sun, and R. Baets, "A novel optical decision circuit based on a Mach-Zehnder or Michelson interferometer and gain clamped semiconductor optical amplifiers," IEEE Photon. Technol. Lett., vol. 10, pp. 1162-1164, August 1998.

[7] O. Kjebon, R. Schatz, S. Lourdudoss, S. Nilsson, B. Stålnacke, and L. Bäckbom, "30 GHz direct modulation bandwidth in detuned loaded InGaAsP DBR-lasers at $1.55 \mathrm{~mm}$ wavelength," Electron. Lett., vol. 33, pp. 488-489, 1997. 